\title{
Publication bias: what is it? How do we measure it? How do we avoid it?
}

This article was published in the following Dove Press journal:

Open Access Journal of Clinical Trials

3 July 2013

Number of times this article has been viewed

\section{Fujian Song \\ Lee Hooper \\ Yoon K Loke}

Norwich Medical School, University of East Anglia, Norwich, UK
Correspondence: Fujian Song Norwich Medical School, Faculty of Medicine and Health Science, University of East Anglia, Norwich Research Park, Norwich, Norfolk NR4 7TJ, UK

Tel 44 I603 59| 253

Email fujian.song@uea.ac.uk
Abstract: Publication bias occurs when results of published studies are systematically different from results of unpublished studies. The term "dissemination bias" has also been recommended to describe all forms of biases in the research-dissemination process, including outcome-reporting bias, time-lag bias, gray-literature bias, full-publication bias, language bias, citation bias, and media-attention bias. We can measure publication bias by comparing the results of published and unpublished studies addressing the same question. Following up cohorts of studies from inception and comparing publication levels in studies with statistically significant or "positive" results suggested greater odds of formal publication in those with such results, compared to those without. Within reviews, funnel plots and related statistical methods can be used to indicate presence or absence of publication bias, although these can be unreliable in many circumstances. Methods of avoiding publication bias, by identifying and including unpublished outcomes and unpublished studies, are discussed and evaluated. These include searching without limiting by outcome, searching prospective trials registers, searching informal sources, including meeting abstracts and $\mathrm{PhD}$ theses, searching regulatory body websites, contacting authors of included studies, and contacting pharmaceutical or medical device companies for further studies. Adding unpublished studies often alters effect sizes, but may not always eliminate publication bias. The compulsory registration of all clinical trials at inception is an important move forward, but it can be difficult for reviewers to access data from unpublished studies located this way. Publication bias may be reduced by journals by publishing high-quality studies regardless of novelty or unexciting results, and by publishing protocols or full-study data sets. No single step can be relied upon to fully overcome the complex actions involved in publication bias, and a multipronged approach is required by researchers, patients, journal editors, peer reviewers, research sponsors, research ethics committees, and regulatory and legislation authorities.

Keywords: publication bias, reporting bias, research-dissemination bias, evidence synthesis, systematic review, meta-analysis

\section{Introduction}

Published literature is the main source of evidence for making clinical and healthpolicy decisions. The number of published studies has increased dramatically over time. ${ }^{1}$ It was estimated that over US\$100 billion investment in biomedical research worldwide generated 1 million research publications per year. ${ }^{2}$ However, there are still concerns that many completed research studies have not been formally published. We may simultaneously have two paradoxical problems: information overload due to an overwhelming volume of literature, and inaccessibility of results from relevant studies. Particularly, if results of published studies are systematically different from those of unpublished studies, published studies will not be a valid representation of 
all studies conducted, and the evidence base for clinical and health-policy decisions will be questionable. ${ }^{3}$

In this article, we provide a comprehensive review of publication bias. We first consider the question about what publication bias is. Then, we discuss methods to detect and avoid publication bias. Our discussion is based on a previous report on publication bias, ${ }^{3}$ complemented and updated with recently published key studies.

\section{What is publication bias? Concepts and definitions}

The term "file-drawer problem" was used by Rosenthal in 1979 to describe the fact that many completed studies have never been published. ${ }^{4}$ Publication of a study in a peerreviewed journal is usually considered as being formally published. It has been reported that about $50 \%$ of completed studies may remain unpublished. ${ }^{3,5,6}$ Publication bias occurs when the publication of studies depends on the nature and direction of the results, so that published studies may be systematically different from those of unpublished studies. ${ }^{7}$ In general, studies with statistically significant or positive results are more likely to be published than those with nonsignificant or negative results. ${ }^{3}$

Publication bias has been recognized as a problem in medical research for many years. The first article with the term "publication bias" that could be identified by searching PubMed was published in 1979 about the association between testicular size and abnormal karyotypes. ${ }^{8}$ Since then, the number of references that are potentially relevant to publication bias has considerably increased (Figure 1).

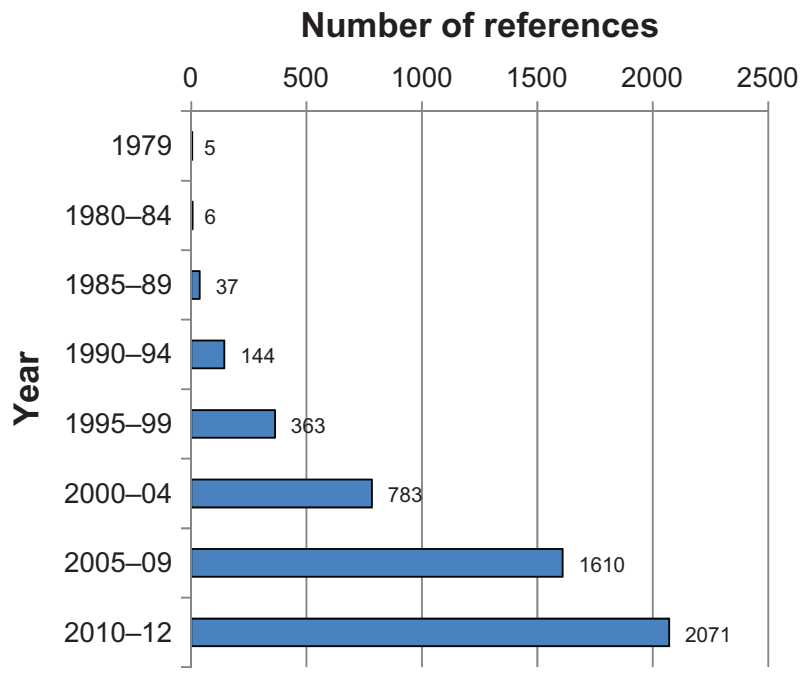

Figure I Number of references relevant to publication bias identified from searching PubMed. Search was conducted on January 28, 2013 using keywords "publication bias" or "reporting bias" or "dissemination bias."
This increase in the number of relevant studies on publication bias may reflect the increased awareness of publication and related biases.

Dissemination of research results is a complex process (Figure 2). In addition to journal papers, study results may be presented at relevant conferences, submitted to medicine regulatory authorities, and in clinical study reports to trial sponsors. ${ }^{3}$ Results presented at conferences are usually only available in abstract form. Clinical study reports contain much more detailed information on methodology and a wider selection of outcomes - fuller results. ${ }^{9}$ Data submitted to regulatory authorities are also important sources of trial results. However, clinical study reports and data submitted to regulatory authorities have limited accessibility, and their use for making evidence-based decisions may not be as straightforward as results published in peer-reviewed journals. Therefore, results of trials that are not published in peer-reviewed journals are conventionally considered as being "unpublished."

The accessibility of trial results depends on whether, when, where, and in what format results of a study are published. ${ }^{10}$ Empirical evidence indicated the existence of biases throughout the whole research-dissemination process, including time-lag bias, outcome-reporting bias, gray-literature bias, full-publication bias, language bias, citation bias, and media-attention bias. ${ }^{3}$ The term "research-dissemination profile" has been recommended to describe the extent to which study results are accessible, which ranges from completely inaccessible to fully accessible. ${ }^{11}$ Dissemination bias occurs when the dissemination profile of research is associated with the direction and/or nature of studies' results. ${ }^{3}$

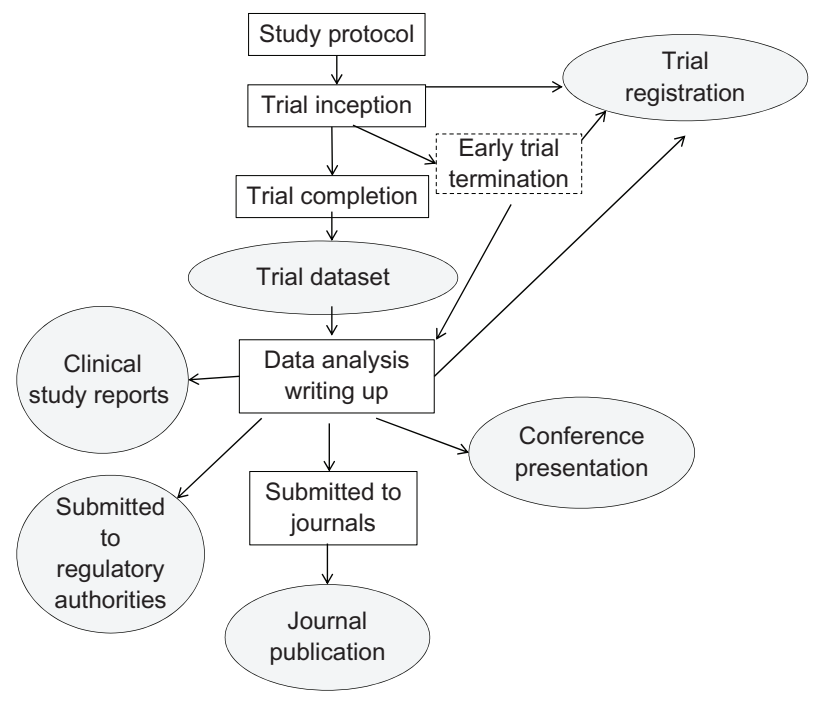

Figure 2 Generation and dissemination of results of clinical trials: a complex process. 
Convincing evidence from recent high-quality empirical studies has confirmed the existence of outcome-reporting bias. ${ }^{12-14}$ Outcome-reporting bias occurs when "positive" outcomes are more likely to be reported than "negative" outcomes in studies with multiple outcomes. Therefore, in contrast to publication bias due to the nonpublication of whole studies, outcome-reporting bias due to selective reporting of outcomes by authors in published studies has been referred to as "within-study publication bias."15 Furthermore, negative results being misinterpreted and reported as positive results in published clinical trials is also prevalent. ${ }^{16,17}$

\section{Causes of publication bias}

Bias may be introduced intentionally or unintentionally, consciously or unconsciously, into the process of research dissemination. ${ }^{3,18}$ The dissemination profile of research may be influenced by investigators, study sponsors, peer reviewers, and journal editors. According to surveys of investigators, the main reasons for nonpublication of completed studies included lack of time or low priority (34.5\%), unimportant results (19.6\%), and journal rejection $(10.2 \%){ }^{3}$ Therefore, the nonpublication of studies was usually due to investigators' failure to write up and submit to journals when the results were considered to be negative or nonsignificant. Publication in peer-reviewed journals is an essential requirement for academic researchers. It is interesting to understand why investigators often failed to submit studies for journal publications. One study found that many researchers may have results from multiple studies that could be publishable, and they usually focused on "wonderful results" and had no time for "negative results." 19 We know that the preparation of manuscripts for journal publication is a time-consuming process. To a certain extent, experienced researchers may be able to predict what results are more likely to be accepted for publication in high-impact journals. Such results may typically be statistically significant, or considered important or positive.

Many clinical studies are sponsored by pharmaceutical companies, and commercial interests may determine the dissemination profile of a study. Many cases have been described in which the publication of studies with negative results has been suppressed by pharmaceutical companies. ${ }^{3,20}$ For example, a company-sponsored study investigated the effect of deferiprone for the prevention of iron toxicity in patients with thalassemia and found that the drug might be harmful. Legal action was taken by the company in order to stop the publication of the results. ${ }^{21,22}$ In many cases, "negative" results submitted to medicinal regulatory authorities have never been published in peer-reviewed journals. ${ }^{23-25}$ Since 2000, the complete suppression of publication of clinical studies may have become more difficult, at least partly due to the public awareness of detrimental consequences of publication bias and the development of trial registration. However, biased selection of outcomes reported in publications is still prevalent. ${ }^{12}$

The publication of non-industry-sponsored studies may also be suppressed or delayed for various reasons. ${ }^{26}$ For example, a large-scale trial of deworming and vitamin A that included one million children in India was completed in 2005 , but was published for the first time many years later in 2013. ${ }^{27}$ The results indicated that the deworming program was not effective in improving weight gain or reducing mortality, which is at odds with policies endorsed by powerful institutions including the World Health Organization, the World Bank, and the Gates Foundation. Therefore, the investigators spent several years checking the trial data to ensure "the credibility of the study."28

\section{Consequences of publication bias}

Publication bias will result in misleading estimates of treatment effects and associations between study variables. Here the consequences of publication bias are considered separately for basic biomedical research, observational studies, and clinical trials.

Results of basic medical research are often used to support subsequent clinical trials. If the results of basic research are falsely positive due to biased selection for publication, ${ }^{29}$ subsequent clinical trials may waste limited resources and fail to confirm the published results of basic studies. ${ }^{30}$ For example, publication bias may be used to explain the observed discrepancy in results between animal studies and clinical trials regarding the neuroprotective efficacy of nicotinamide for focal cerebral ischemia. ${ }^{31}$

Results of observational studies are often highly contradictory over a wide range of health risk factors, ${ }^{32}$ which may be partly due to publication bias. For example, publication bias may cause highly contradictory results observed in early published studies of genetic associations. ${ }^{33}$

Publication bias in clinical trials has a direct impact on patients' and populations' health. When the relative efficacy of a treatment is overestimated because of publication bias, health resources can be wasted by purchasing more expensive interventions, instead of cheaper alternatives, without corresponding improvement in outcome. There are also many reported cases in which patients have received ineffective or harmful treatments. ${ }^{3}$ For example, biased reporting of trial 
results delayed the detection of increased mortality risk of rofecoxib for Alzheimer's disease and cognitive impairment, and more than 80 million patients had used rofecoxib before its withdrawal in 2004. ${ }^{34}$

\section{How do we measure publication bias?}

The observation of disproportionately frequent positive findings in published studies and larger effect sizes in smaller studies could be used indirectly to estimate the extent of publication bias. More convincing evidence on publication bias was from studies that compared published and unpublished studies, and studies that followed up cohorts of studies from their inception.

\section{High proportion of positive findings in published studies}

Sterling in 1959 suspected that nonsignificant results might be underreported because the results of $97 \%$ of psychological studies published in four journals were statistically significant, ${ }^{35}$ and the same author in 1995 concluded that the practices leading to publication bias in psychological research had not changed over a period of 30 years. ${ }^{36}$ The reported proportion of published studies with positive outcomes was $80 \%$ in emergency or general medicine, ${ }^{37} 53 \%-72 \%$ of trials of anesthesiology, ${ }^{38} 82 \%$ in dental journals, ${ }^{39}$ and 50\%-76\% in studies of complementary and alternative medicine. ${ }^{40}$ However, the proportion of significant results in published studies tells us nothing about the proportion of significant results in unpublished studies.

\section{Comparing published with unpublished studies}

Publication bias can be directly detected by comparing published results and unpublished results from studies that investigate the same research question. If both published and unpublished results are available in a meta-analysis, the difference between the two could be compared to estimate the extent of publication bias. For example, data from published trials of selective serotonin-reuptake inhibitors indicated favorable risk-benefit profile for children with depression, but data from unpublished trials of paroxetine, sertraline, and citalopram suggested that risks could outweigh benefits in children and young people. ${ }^{41}$ However, it is usually impossible to be certain about whether there are any unpublished results and how many remain unpublished. Even if we know of the existence of unpublished results, it is still very difficult, or even impossible, to obtain unpublished data.

\section{Following up cohorts of studies}

A cohort of studies can be tracked from before their formal publication and the odds of publication compared between studies with different results. For example, studies approved by a research ethics committee or institutional review board were followed up to investigate the extent of publication bias. ${ }^{42,43}$

A systematic review of publication bias included studies that tracked a cohort of studies and reported the rate of publication by results. ${ }^{44}$ The included cohort studies were separated into four categories: inception cohorts that followed up a cohort of studies from the beginning, regulatory cohorts that followed up studies submitted to regulatory authorities, abstract cohorts that investigated the full publication of meeting abstracts, and manuscript cohorts that reported the publication of manuscripts submitted to journals. In this meta-analysis, study results were classified as "statistically significant" versus "nonsignificant," and "positive" versus "nonpositive." Positive results may have been defined differently in studies of publication bias, including "significant," "favorable," "important," or "confirmatory" results. The meta-analysis results confirmed that studies with statistically significant or positive results were more likely to be formally published than those with nonsignificant or nonpositive results (Figure 3 ). The meta-analysis results also suggested that the biased selection for publication by

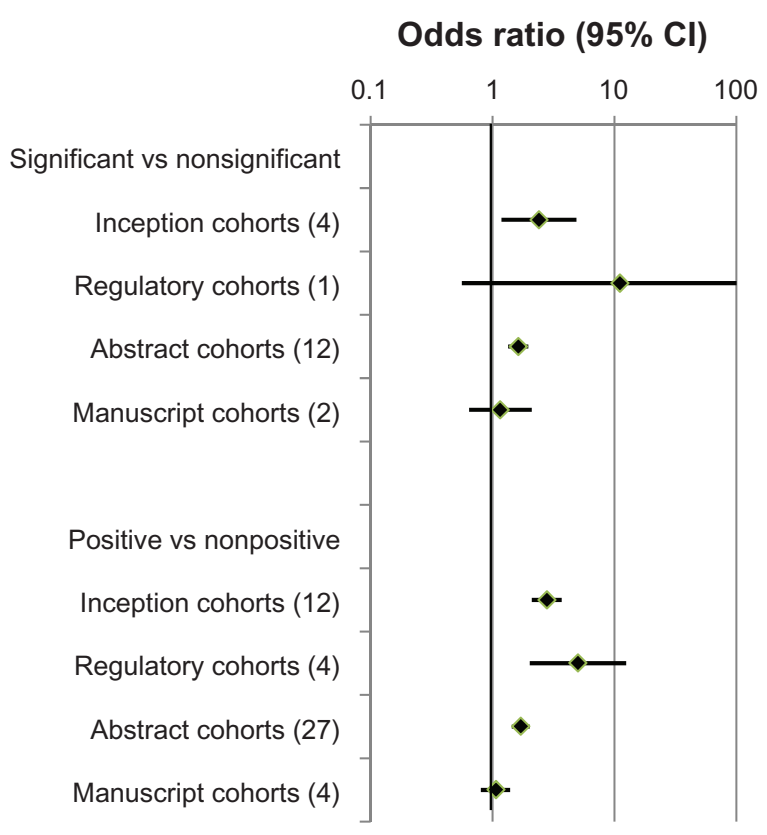

Figure 3 Results of a meta-analysis of cohort studies on publication bias. Odds ratio $>$ I indicates that studies with statistically significant or positive results were more likely to be formally published than those with nonsignificant or nonpositive results (data from Song et al $2009^{44}$ ).

Abbreviation: $\mathrm{Cl}$, confidence interval. 
results occurred before the submission of manuscripts to journals. ${ }^{44}$

Cohorts of trial protocols were also used to estimate the extent of outcome-reporting bias. Chan and colleagues compared reported outcomes and unreported outcomes by following up a cohort of 102 protocols of randomized controlled trials (RCTs) approved by the Danish Research Ethics Committee from 1994 to $1995^{12}$ and a cohort of 48 RCT protocols approved by the Canadian Institutes of Health Research from 1990 to $1998 .{ }^{13}$ They found that $31 \%-50 \%$ of efficacy outcomes and $59 \%-65 \%$ of harm outcomes were incompletely reported, and statistically nonsignificant outcomes were more likely to be incompletely reported than statistically significant outcomes. ${ }^{12,13}$

\section{Funnel plot and related methods}

Because smaller studies have larger random errors, the results of smaller studies are more widely spread around the average estimate compared to the results of larger studies. If there is no publication bias, a plot of sample size against estimated effect size from primary studies in a meta-analysis should be shaped like a funnel (Figure 4).

Light and Pillemer in 1984 recommended that a funnel plot could be used to estimate the risk of publication bias in meta-analyses. ${ }^{45}$ When the true treatment effect equals zero, the biased selection of studies with significant results will produce a funnel plot with an empty area around zero (Figure 5A). When the true effect size is small or moderate, the funnel plot may become asymmetric because of publication bias (Figure 5B). The use of funnel plots for detecting publication bias is also based on an assumption that larger-scale studies are less vulnerable to publication bias than smaller studies.

Many statistical methods have been developed to test funnel-plot asymmetry. It is hoped that statistical methods

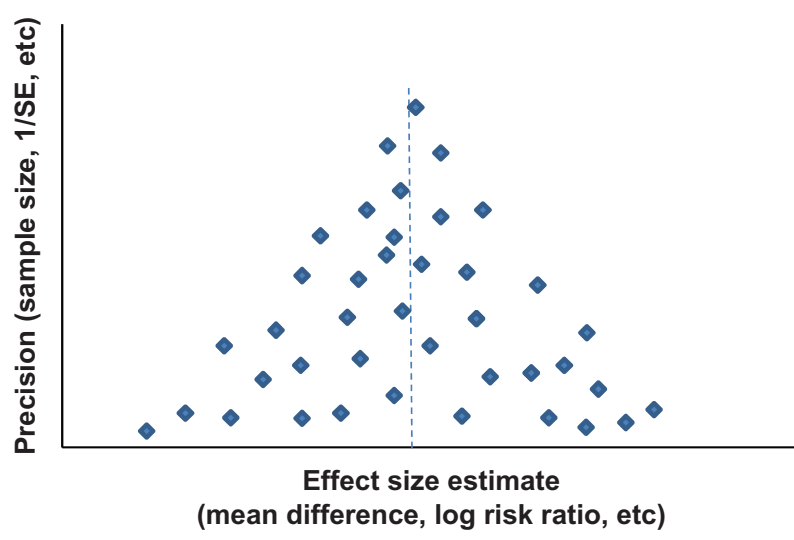

Figure 4 Funnel-plot illustration with sufficient number of nonbiased studies. Each point represents a study in a meta-analysis.
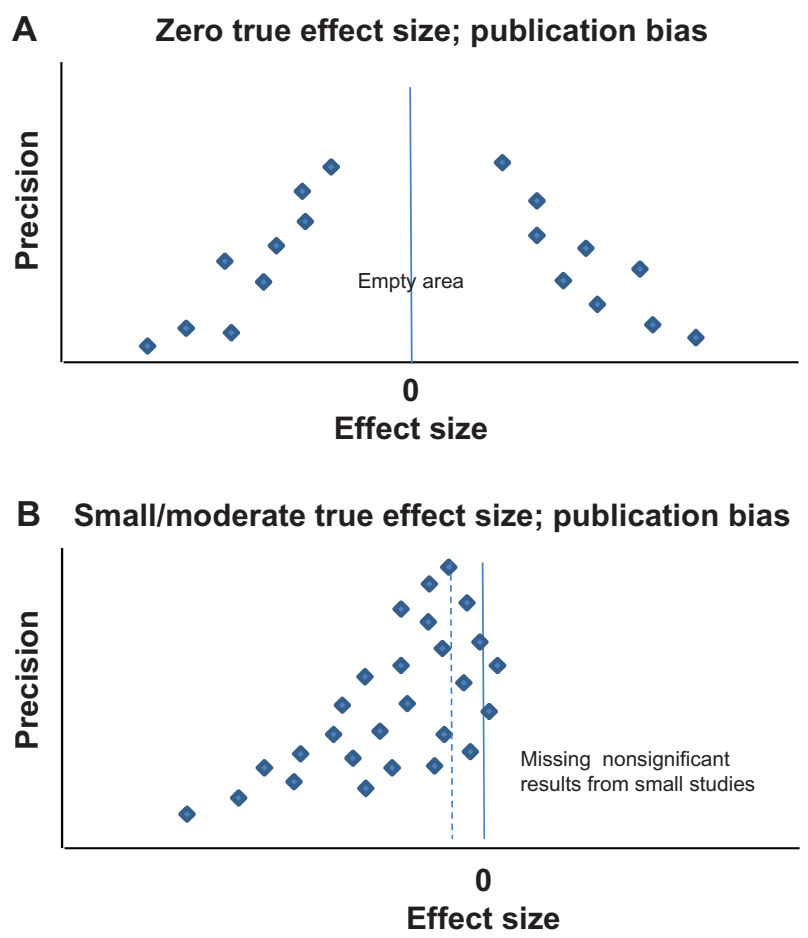

Figure $\mathbf{5}$ (A and $\mathbf{B}$ ) The possible impact of publication bias on the shape of funnel plots. (A) When the true effect size is zero; (B) when the true effect size is small or moderate.

may provide a more objective and accurate assessment of funnel-plot asymmetry than subjective visual assessment. However, all available statistical tests have important limitations, and different statistical methods often lead to different conclusions about funnel-plot asymmetry. ${ }^{3}$ The performance of tests for funnel-plot asymmetry is particularly poor when the number of studies is small and heterogeneity is large in meta-analysis. ${ }^{46}$ It has been recommended that the tests for funnel-plot asymmetry should not be used in meta-analyses that include fewer than ten studies. ${ }^{47}$

There are many important limitations of funnel plots for detecting publication bias. A sufficiently large number of studies with varying sizes is required, and the shape of a funnel plot may be subject to different interpretations. Because of the relatively small number of studies in typical meta-analyses, statistical tests may fail to detect funnel-plot asymmetry even if it exists. Most importantly, a skewed funnel plot may be caused by factors other than publication bias, including clinically meaningful heterogeneity, poor methodological design of small studies, inadequate analysis, fraud, choice of effect measures, and chance. ${ }^{48}$ An asymmetric funnel plot may reveal "small-study effects" by indicating that smaller studies tend to report larger effect sizes. However, the observed "small-study effects" may not necessarily be caused by publication bias. ${ }^{47}$ For example, 
data from a systematic review of calcium supplementation for the prevention of pr-eclampsia ${ }^{49}$ could be used to produce a significantly asymmetric funnel plot (Figure 6). However, this asymmetric funnel plot may be at least partly explained by different baseline risk of hypertension and differences in dietary calcium intake between smaller and larger studies in this systematic review. ${ }^{49}$

\section{How to avoid publication bias?}

There are a number of steps that may be taken to reduce the risk of publication bias. The choice of strategy depends on whether the aim is to tackle entire sets of missing studies, or whether selective/incomplete reporting of data by authors is considered to be the primary problem. Equally, unavailability of large chunks of data may arise from journal or editorial policies regarding novelty of the manuscript as well as restrictions on page length of printed articles. Hence, any attempts to reduce the overall burden of publication bias will have to adopt a multipronged approach that includes trial registration and changes in the current publication model.

\section{Identifying and including unpublished studies}

Locating unpublished studies and unpublished outcomes of published studies for inclusion within a systematic review may help to reduce publication bias, providing a better estimate of effectiveness or association. Detailed advice on searching for both published and unpublished studies can be found in the Cochrane handbook. ${ }^{50}$ Searching for unpublished studies increased from $35 \%$ of efficacy reviews in 1996 to $61 \%$ in $2006 .{ }^{51}$ However, locating unpublished

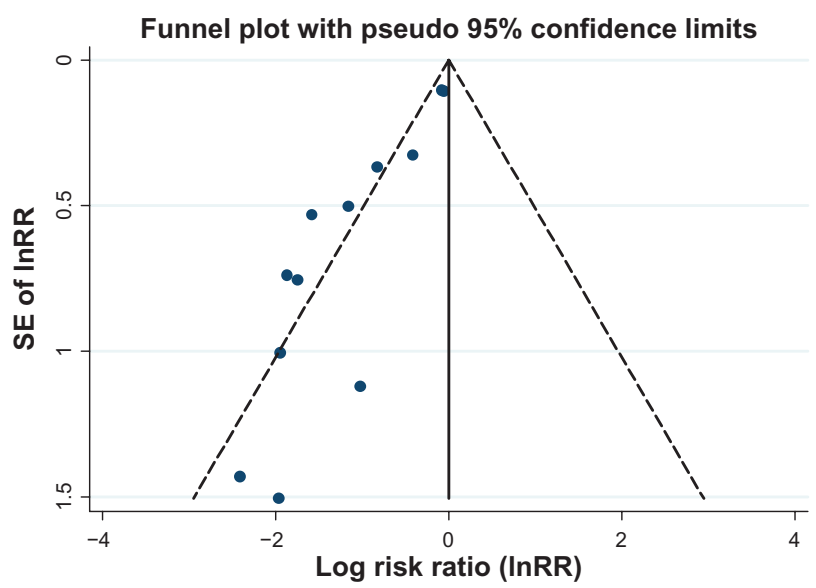

Figure 6 An example of a funnel plot of risk ratio (on log scale) against inverse of standard error, data from Hofmeyr et al, 2006. ${ }^{49}$ Statistical testing of funnelplot asymmetry: Peters test, $P=0.009$, Harbord's test, $P=0.000$ (by using STATA "metabias" command). studies can be difficult and time-consuming. It is never clear that all unpublished studies have been located, and it may be impossible to access data from an unpublished study even if its existence is discovered. Additionally, the discovered studies may not be representative of the results or biases of the full set of unpublished studies.

A basic strategy that can be used within systematic reviews to locate missing or unpublished outcomes of published studies is to conduct electronic searches without limiting by outcome terms. This is a good strategy in any case, as outcomes not found to be statistically significant are often not reported in abstracts or represented by indexing $(\mathrm{MeSH})$ terms, even when those outcomes are reported in full in the main text of the paper. ${ }^{50}$ To find unpublished outcomes, studies that appear appropriate in terms of their population, intervention (or exposure), comparison, and methodology are collected as full text regardless of outcomes reported, provisionally included, and study authors contacted to provide details of their outcomes (or any review outcomes additional to those provided in the published paper). Where trial-registration details are found, this can help reviewers to know when unpublished outcomes exist, and to ask for them with more authority, but it can be productive to ask even without this information. In a recent systematic review of RCTs, we established contact with authors of 19 trials, and included additional outcomes for twelve studies (of the 42 included RCTs). ${ }^{52}$ However a further twelve trials had clearly measured but not reported at least one outcome of interest for our review (or reported insufficiently to use in the meta-analysis, eg, by saying that there was no effect of the intervention on an outcome, without providing numerical data, or omitting variance data).

Methods for locating unpublished studies also need to be built into reviews. These can include searching trial registers for completed and ongoing studies (see the next section), searching informal publication sources including meeting abstracts (included within Cochrane trial registers) and $\mathrm{PhD}$ theses, searching regulatory bodies (such as the US Food and Drug Administration (FDA) database), contacting the authors of included studies, and contacting pharmaceutical or medical device companies for further studies. Data retrieved this way can be in the form of complete manuscripts, simple data tables, answers to specific queries, study protocols, and/or full data sets from completed studies. One Cochrane review was updated, including highly detailed FDA regulatory comments and reviews that allowed the researchers to include data from 25 studies, but they found they could not use data from a further 42 studies where discrepancies in the regulatory 
data were unresolved, or insufficient data were provided by pharmaceutical companies that conducted the trials. ${ }^{53}$ Hart et al added unpublished FDA data to already-conducted meta-analyses and found that the additional studies reduced the effect size in $46 \%$ of the 42 outcomes. ${ }^{54}$

While searching for and including unpublished studies and outcomes in systematic reviews is probably important in reducing if not eliminating publication bias, there have been suggestions that in some circumstances, including unpublished studies may increase publication bias. Ferguson and Brannick examined 91 meta-analyses of psychological interventions, and found that while $63 \%$ had tried to find unpublished studies, inclusion of unpublished studies did not reduce the likelihood of publication bias, and even increased it. ${ }^{55}$ They suggested that this might be due to selection bias in unpublished studies, resulting from review authors being overrepresented as study authors of the unpublished trials. However, the methodology of this analysis has been challenged on several counts: their definition of unpublished studies was unusual. Additionally, their analyses found increased heterogeneity when "unpublished" studies were included, and they interpreted this as an increase in bias, but this may simply be confirmation that unpublished studies provide different effect sizes than published studies. ${ }^{56}$

Another important mechanism for reducing the impact of publication bias is to keep systematic reviews regularly updated. This is useful, as it ensures that the results of studies whose publication is delayed (often due to having less favorable results) and later larger trials are built into the review. ${ }^{3}$ An analysis of unpublished studies within Cochrane reviews found that $38 \%$ of unpublished studies were eventually published, but their earlier inclusion would have reduced time-lag bias. ${ }^{57}$

\section{Prospective registration of trials and its limitations}

Trial registration is a process by which details about the design and conduct of a clinical trial are published (in an agreed format) in a publicly available resource, which typically is a website registry, ideally before study recruitment begins. A wide range of international trial registries can be searched through a single portal, such as the metaRegister of Controlled Trials (http://www.controlled-trials. com $/ \mathrm{mrct} / \mathrm{mrct}$ about). Trial registration is considered to have both scientific and ethical implications, particularly in light of item 19 in the Declaration of Helsinki, which states "Every clinical trial must be registered in a publicly accessible database before recruitment of the first subject" (http://www.wma.net/en/30publications/10policies/b3/ index.html). The WHO recognizes the importance of trial registration in enabling health care decisions to be based on a collection of all available evidence (http://www.who.int/ ictrp/trial reg/en/index.html). Other potential benefits of registration include the ability to identify gaps while avoiding duplication of research, and the facilitation of participant recruitment as well as collaboration amongst researchers working in the same area. In theory, trial registration should reduce the possibility of entire studies disappearing off the radar, or of never emerging in the first place. However, it must be recognized that there is no foolproof method of rigorously policing and enforcing registration, and there are a number of other issues and limitations that have subsequently been reported in empirical evaluations. Here, it is also worth noting that the initial aims of the trial registries were to enable easy identification of trials, and there were no specific requirements for detailed descriptions of methods, statistical techniques, or outcomes.

The potential lack of methodological detail in registered trials has been assessed recently by Reveiz et al, who compiled a cohort of 265 ongoing RCTs from seven registries accessed through the WHO portal. ${ }^{58}$ Using the components from the Cochrane risk-of-bias tool, the researchers found that key items related to randomization, ie, sequence generation or allocation concealment was seldom detailed. Reveiz et al identified major deficiencies in three areas, with the majority of registered trials failing to report useful methodological information on allocation concealment (97.9\%) and harm (89.5\%), as well as lacking detail regarding method of blinding (86.2\%, after excluding open-label RCTs). Conversely, other aspects, such as eligibility criteria, primary outcomes, and duration of follow-up, were reported more frequently. This may in some respects be related to the fields for data entry stipulated by individual trial registries, with the Australasian and Indian registries showing higher proportions of adequate reporting. While methodological details may not be mandatory, the availability of such details allows readers to appraise trial validity fully and to judge the possibility of bias affecting the results.

It could be argued that additional details and results from the registered trial would be accessible in journal manuscripts or through direct contact with the registered investigators. However, Ross et al found that in a sample of 677 completed trials, 311 (46\%) had been published and indexed within Medline, but of these, only 96 (31\%) gave a citation within clinicaltrials.gov of a relevant published article.$^{59}$ In the sample of $>300$ trials with no identifiable 
publication (despite more than 2 years elapsing since date of trial completion), details of study investigators were available in only 117 instances, and attempts to contact the investigators yielded no response from 73 trials, or a response that no publication was available in 40 cases. These findings have been corroborated by Bourgeois et al who demonstrated that 184 of $546(33.7 \%)$ registered drug trials did not have published results. ${ }^{60}$ Hence, while registration procedures may have helped facilitate more rapid identification of trials, there remains a problem with accessing the results (either through direct contact with investigators or through the registry/bibliographic database), as well as the lack of dissemination of trial findings. This problem may prove particularly irksome to systematic reviewers who are able to identify relevant trials from registries, but may have no means of sourcing the results for their data synthesis.

Systematic reviewers also have to face the challenge of making a link between the trial-registration record and a journal article. If trial-registration numbers are not rigorously reported within the associated journal publications, systematic reviewers may run the risk of including certain studies twice. A study from the Dutch Cochrane Centre found that trial-registration numbers were described in only $166 / 302$ (55\%) of the RCTs published in November 2010 that were indexed in Medline. ${ }^{61}$ Moreover, 39\% of the published RCTs did not appear to have been registered at all. This creates difficulties for reviewers who are attempting to check for certain elements of selective outcome reporting by comparing registry listings against the subsequent journal publication.

There have also been mixed reports of success in using trial registries to detect outcome-reporting biases in published articles. Hannink et al looked at 152 RCTs published between 2007 and 2012 of surgical interventions with prespecified primary outcomes, and found that 75/152 (49\%) had some discrepancy between registered outcomes and the published data. ${ }^{62}$ These seemed to involve the omission or introduction of a primary outcome, and may have been related to a bias towards reporting statistically significant results in $28 \%$ of the papers. Huic et al looked at a different sample of 152 RCTs and found that the registry entries commonly failed to provide adequate description of key secondary outcomes (44.1\% of RCTs) or primary outcomes (38.8\% of RCTs). ${ }^{63}$ Prevalence of differences between the registry entry and the journal publication varied depending on the data field of interest, ranging from $13.8 \%$ to $77.6 \%$. However, in a more recent study, Norris et al reported a catalog of problems during their attempts to evaluate selective outcome or analysis reporting through comparison of registry entries and journal reports of trials that had been included in systematic reviews of effectiveness. ${ }^{64}$ Key limitations included the aforementioned lack of detail in specification of outcome, as well as trial registration taking place after completion of studies (between $25 \%$ and $50 \%$ of the time in this cohort) and unavailability of results in the clinicaltrials.gov registry. In particular, Norris et al found it difficult to judge whether certain new outcomes/analyses found within the journal publications had been added post hoc, or whether these were genuinely prespecified analyses that had inadvertently been missed due to poor data entry in the trial registry. ${ }^{64}$

\section{Open-access policy and changes in publication process}

While an open-access policy allows free public access to journal articles (either at time of publication or at some point 6-12 months thereafter), this in itself may have no impact on selective publication of results. However, publication bias could be reduced if journal editors moved away from the policy of giving greater priority to articles that were subjectively perceived as having greater novelty or importance, or significant findings. Some open access journals (such as PLoS One) focus on scientific and technical quality of the manuscript rather than novelty, and this may encourage authors to submit reports of negative or unexciting results. Equally, there is some value from a commitment by certain industry sponsors to provide unrestricted access (via Internet registries) to aggregated data for all their clinical trials within a stipulated interval after study completion. ${ }^{65}$ It is not clear whether the exact format and comprehensiveness of such data will match or exceed that provided within journal manuscripts. Finally, journal editors can give greater weight to transparency by requiring trial protocols to be published as appendices alongside the trial report. Intriguingly, the British Medical Journal has taken the even more dramatic step of requiring investigators to submit full data sets to accompany trials that are published in that journal. ${ }^{65}$ Again, it remains to be seen what detail and depth will be available within such data sets, or whether there will be a substantial drop in submissions to that journal.

Reporting bias will be avoided if the full data from clinical trials can be accessible to researchers and systematic reviewers, which will have considerable benefits for patients, society, and medical science. However, national and international legislation is required in order to achieve data-sharing in clinical research. ${ }^{66}$

\section{Summary and conclusion}

Publication bias occurs when results of published studies are systematically different from results of unpublished studies. 
The accessibility of trial results depends on whether, when, where, and in what format results of a study are published. The term "research-dissemination profile" can be used to describe the extent to which study results are accessible, which ranges from completely inaccessible to fully accessible. Dissemination bias occurs when the dissemination profile of research is associated with the direction and/or nature of its results.

Empirical evidence indicates the existence of biases throughout the whole research-dissemination process, including time-lag bias, outcome-reporting bias, grayliterature bias, full-publication bias, language bias, and citation bias. Biased selection for research dissemination is related to the commercial and noncommercial interests of investigators, research sponsors, peer reviewers, and journal editors. Consequences of research-dissemination bias may depend on types of research (basic biomedical, observational, or clinical studies) and levels of result acceptability. The detrimental consequences of publication bias include avoidable suffering of patients and waste of limited resources.

The extent of publication bias could be directly measured by comparing the results of published and unpublished studies, and by comparing the rate of publication of studies with different results. More often, we may have to use indirect approaches to estimate the risk of publication bias in published studies, including the proportion of positive findings in published studies or funnel plot and related methods. It is particularly important to recognize that publication bias is only one of many possible explanations for an asymmetric funnel plot in meta-analysis.

Locating unpublished studies and unpublished outcomes of published studies for inclusion within a systematic review may provide a less biased estimate of effectiveness or association. The compulsory registration of all trials at inception is an important development in preventing publication bias. Since publication bias may creep in at various points from decision to submit to a trial registry, selection of outcomes for analysis, preparation for submission, and journal editorial processes, no single step can be relied upon to overcome fully the complex actions involved in publication bias, and a multipronged approach is required. Given the potential ethical and clinical implications of nonpublication/selective reporting of trial results, it would certainly help if ethical approval was granted conditional on full disclosure at a later stage. During the consent process, patients and healthy participants who volunteer in good faith to enter clinical trials also need to be informed explicitly whether the findings from the trial will or will not be made available on an unrestricted (but fully anonymized) basis.

\section{Acknowledgments}

We received no specific funding for this review article. The views expressed in this article are those of the authors.

\section{Disclosure}

The authors report no conflicts of interest in this work.

\section{References}

1. Bastian H, Glasziou P, Chalmers I. Seventy-five trials and eleven systematic reviews a day: how will we ever keep up? PLoS Med. 2010;7(9):e1000326.

2. Chalmers I, Glasziou P. Avoidable waste in the production and reporting of research evidence. Lancet. 2009;374(9683):86-89.

3. Song F, Parekh S, Hooper L, et al. Dissemination and publication of research findings: an updated review of related biases. Health Technol Assess. 2010;14(8):iii, ix-xi, 1-193.

4. Rosenthal R. The "file drawer problem" and tolerance for null results. Psychol Bull. 1979;86(3):638-641.

5. Dwan K, Gamble C, Kolamunnage-Dona R, Mohammed S, Powell C, Williamson PR. Assessing the potential for outcome reporting bias in a review: a tutorial. Trials. 2010;11:52.

6. Scherer RW, Langenberg P, von Elm E. Full publication of results initially presented in abstracts. Cochrane Database Syst Rev. 2007;(2): MR000005.

7. Dickersin K. The existence of publication bias and risk factors for its occurrence. JAMA. 1990;263(10):1385-1389.

8. Boisen E. Testicular size and shape of 47, XYY and 47, XXY men in a double-blind, double-matched population survey. Am J Hum Genet. 1979;31(6):697-703.

9. Doshi P, Jones M, Jefferson T. Rethinking credible evidence synthesis. BMJ. 2012;344:d7898.

10. Main J, Reddy L, Lazarevic M, Whelan PJ. Are late-onset eating disorders in the elderly really the more common variant? Concerns around publication bias. Int Psychogeriatr. 2011;23(4):670-671.

11. Song F, Eastwood AJ, Gilbody S, Duley L, Sutton AJ. Publication and related biases. Health Technol Assess. 2000;4(10):1-115.

12. Chan AW, Hrobjartsson A, Haahr MT, Gotzsche PC, Altman DG. Empirical evidence for selective reporting of outcomes in randomized trials: comparison of protocols to published articles. JAMA. 2004;291(20):2457-2465.

13. Chan AW, Krleza-Jerić K, Schmid I, Altman DG. Outcome reporting bias in randomized trials funded by the Canadian Institutes of Health Research. CMAJ. 2004;171(7):735-740.

14. Kirkham JJ, Dwan KM, Altman DG, et al. The impact of outcome reporting bias in randomised controlled trials on a cohort of systematic reviews. $B M J .2010 ; 340: \mathrm{c} 365$.

15. Higgins J, Altman DG. Assessing risk of bias in included studies. In: Higgins J, Green S, editors. Cochrane Handbook for Systematic Reviews of Interventions. Version 5.0.0. Oxford: Cochrane Collaboration; 2008

16. Vera-Badillo FE, Shapiro R, Ocana A, Amir E, Tannock IF. Bias in reporting of end points of efficacy and toxicity in randomized, clinical trials for women with breast cancer. Ann Oncol. 2013;24(5): 1238-1244.

17. You B, Gan HK, Pond G, Chen EX. Consistency in the analysis and reporting of primary end points in oncology randomized controlled trials from registration to publication: a systematic review. J Clin Oncol. 2012;30(2):210-216.

18. Bax L, Moons KG. Beyond publication bias. J Clin Epidemiol. 2011;64(5):459-462.

19. Calnan M, Smith GD, Sterne JAC. The publication process itself was the major cause of publication bias in genetic epidemiology. $J$ Clin Epidemiol. 2006;59(12):1312-1318. 
20. McGauran N, Wieseler B, Kreis J, Schuler YB, Kölsch H, Kaiser T. Reporting bias in medical research - a narrative review. Trials. 2010;11:37.

21. Nathan DG, Weatherall DJ. Academia and industry: lessons from the unfortunate events in Toronto. Lancet. 1999;353(9155):771-772.

22. Nathan DG, Weatherall DJ. Academic freedom in clinical research. N Engl J Med. 2002;347(17):1368-1371.

23. Panahloo Z. Data on neuraminidase inhibitors were made available. BMJ. 2004;328(7438):523.

24. van Heteren $\mathrm{G}$. Wyeth suppresses research on pill, programme claims. BMJ. 2001;322(7286):571.

25. Turner EH, Matthews AM, Linardatos E, Tell RA, Rosenthal R. Selective publication of antidepressant trials and its influence on apparent efficacy. $N$ Engl J Med. 2008;358(3):252-260.

26. Godlee F. Timely publication of all trial results may mean less overtreatment. BMJ. 2013;346:f159.

27. Awasthi S, Peto R, Read S, Richards SM, Pande V, Bundy D. Population deworming every 6 months with albendazole in 1 million pre-school children in north India: DEVTA, a cluster-randomised trial. Lancet. 2013;381(9876):1478-1486.

28. Hawkes N. Deworming debunked. BMJ. 2013;346:e8558.

29. ter Riet G, Korevaar DA, Leenaars M, et al. Publication bias in laboratory animal research: a survey on magnitude, drivers, consequences and potential solutions. Plos One. 2012;7(9):e43404.

30. Perel P, Roberts I, Sena E, et al. Comparison of treatment effects between animal experiments and clinical trials: systematic review. BMJ. 2007;334(7586):197.

31. Macleod MR, O'Collins T, Howells DW, Donnan GA. Pooling of animal experimental data reveals influence of study design and publication bias. Stroke. 2004;35(5):1203-1208.

32. Taubes G. Epidemiology faces its limits. Science. 1995;269(5221): 164-169.

33. Ioannidis JP, Trikalinos TA. Early extreme contradictory estimates may appear in published research: the Proteus phenomenon in molecular genetics research and randomized trials. J Clin Epidemiol. 2005;58(6): 543-549.

34. Psaty BM, Kronmal RA. Reporting mortality findings in trials of rofecoxib for Alzheimer disease or cognitive impairment: a case study based on documents from rofecoxib litigation. JAMA. 2008;299(15): 1813-1817.

35. Sterling T. Publication decisions and their possible effects on inferences drawn from tests of significance - or vice versa. J Am Stat Assoc. 1959;54(285):30-34.

36. Sterling TD, Rosenbaum WL, Weinkam JJ. Publication decisions revisited - the effect of the outcome of statistical tests on the decision to publish and vice-versa. Am Stat. 1995;49(1):108-112.

37. Moscati R, Jehle D, Ellis D, Fiorello A, Landi M. Positive-outcome bias: comparison of emergency medicine and general medicine literatures. Acad Emerg Med. 1994;1(3):267-271.

38. De Oliveira GS Jr, Chang R, Kendall MC, Fitzgerald PC, McCarthy RJ. Publication bias in the anesthesiology literature. Anesth Analg. 2012;114(5):1042-1048.

39. Yuan JC, Shyamsunder N, Ricardo Barao VA, Lee DJ, Sukotjo C. Publication bias in five dental implant journals: an observation from 2005 to 2009. Int J Oral Maxillofac Implants. 2011;26(5):1024-1032.

40. Sood A, Knudsen K, Sood R, et al. Publication bias for CAM trials in the highest impact factor medicine journals is partly due to geographical bias. J Clin Epidemiol. 2007;60(11):1123-1126.

41. Whittington CJ, Kendall T, Fonagy P, Cottrell D, Cotgrove A, Boddington E. Selective serotonin reuptake inhibitors in childhood depression: systematic review of published versus unpublished data. Lancet. 2004;363(9418):1341-1345.

42. Easterbrook PJ, Berlin JA, Gopalan R, Matthews DR. Publication bias in clinical research. Lancet. 1991;337(8746):867-872.

43. Dickersin K, Min YI, Meinert CL. Factors influencing publication of research results. Follow up of applications submitted to two institutional review boards. JAMA. 1992;267(3):374-378.
44. Song F, Parekh-Bhurke S, Hooper L, et al. Extent of publication bias in different categories of research cohorts: a meta-analysis of empirical studies. BMC Med Res Methodol. 2009;9:79.

45. Light RJ, Pillemer DB. Summing Up: The Science of Reviewing Research. Cambridge: Harvard University Press; 1984.

46. Ioannidis JPA, Trikalinos TA. The appropriateness of asymmetry tests for publication bias in meta-analyses: a large survey. CMAJ. 2007;176(8):1091-1096.

47. Sterne J, Egger M, Moher D. Addressing reporting biases. In: Higgins J, Green S, editors. Cochrane Handbook for Systematic Reviews of Interventions. Version 5.0.0. Oxford: Cochrane Collaboration; 2008.

48. Egger M, Davey Smith G, Schneider M, Minder C. Bias in meta-analysis detected by a simple, graphical test. BMJ. 1997;315(7109):629-634.

49. Hofmeyr GJ, Atallah AN, Duley L. Calcium supplementation during pregnancy for preventing hypertensive disorders and related problems. Cochrane Database Syst Rev. 2006;(3):CD001059.

50. Lefebvre C, Manheimer E, Glanville J. Searching for studies. In: Higgins J, Green S, editors. Cochrane Handbook for Systematic Reviews of Interventions. Version 5.0.0. Oxford: Cochrane Collaboration; 2008.

51. Parekh-Bhurke S, Kwok CS, Pang C, et al. Uptake of methods to deal with publication bias in systematic reviews has increased over time, but there is still much scope for improvement. J Clin Epidemiol. 2011;64(4):349-357.

52. Hooper L, Kay C, Abdelhamid A, et al. Effects of chocolate, cocoa, and flavan-3-ols on cardiovascular health: a systematic review and meta-analysis of randomized trials. Am J Clin Nutr. 2012;95(3): 740-751.

53. Jefferson T, Jones MA, Doshi P, et al. Neuraminidase inhibitors for preventing and treating influenza in healthy adults and children. Cochrane Database Syst Rev. 2012;1:CD008965.

54. Hart B, Lundh A, Bero L. Effect of reporting bias on meta-analyses of drug trials: reanalysis of meta-analyses. BMJ. 2012;344:d7202.

55. Ferguson CJ, Brannick MT. Publication bias in psychological science: prevalence, methods for identifying and controlling, and implications for the use of meta-analyses. Psychol Methods. 2012;17(1):120-128.

56. Rothstein HR, Bushman BJ. Publication bias in psychological science: comment on Ferguson and Brannick (2012). Psychol Methods. 2012;17(1): 129-136.

57. van Driel ML, De Sutter A, De Maeseneer J, Christiaens T. Searching for unpublished trials in Cochrane reviews may not be worth the effort. $J$ Clin Epidemiol. 2009;62(8):838-844.

58. Reveiz L, Chan AW, Krleza-Jeric K, et al. Reporting of methodologic information on trial registries for quality assessment: a study of trial records retrieved from the WHO search portal. PLoS One. 2010;5(8):e12484.

59. Ross JS, Mulvey GK, Hines EM, Nissen SE, Krumholz HM. Trial publication after registration in ClinicalTrials.Gov: a cross-sectional analysis. PLoS Med. 2009;6(9):e1000144.

60. Bourgeois FT, Murthy S, Mandl KD. Outcome reporting among drug trials registered in ClinicalTrials.gov. Ann Intern Med. 2010;153(3): $158-166$.

61. van de Wetering FT, Scholten RJ, Haring T, Clarke M, Hooft L. Trial registration numbers are underreported in biomedical publications. PLoS One. 2012;7(11):e49599.

62. Hannink G, Gooszen HG, Rovers MM. Comparison of registered and published primary outcomes in randomized clinical trials of surgical interventions. Ann Surg. 2013;257(5):818-823.

63. Huic M, Marusic M, Marusic A. Completeness and changes in registered data and reporting bias of randomized controlled trials in ICMJE journals after trial registration policy. PLoS One. 2011;6(9):e25258.

64. Norris SL, Holmer HK, Ogden LA, et al. Selective Outcome Reporting as a Source of Bias in Reviews of Comparative Effectiveness. Rockville (MD): Agency for Healthcare Research and Quality; 2012.

65. Godlee F. Clinical trial data for all drugs in current use. BMJ. 2012;345:e7304.

66. Gotzsche PC. Why we need easy access to all data from all clinical trials and how to accomplish it. Trials. 2011;12:249. 
Open Access Journal of Clinical Trials

Dovepress

\section{Publish your work in this journal}

The Open Access Journal of Clinical Trials is an international, peerreviewed, open access journal publishing original research, reports, editorials, reviews and commentaries on all aspects of clinical tria design, management, legal, ethical and regulatory issues, case record form design, data collection, quality assurance and data auditing methodologies. The manuscript management system is completely online and includes a very quick and fair peer-review system, which is all easy to use. Visit http://www.dovepress.com/testimonials.php to read real quotes from published authors.

Submit your manuscript here: http://www.dovepress.com/open-access-journal-of-clinical-trials-journal 